\title{
Virtual Network Mapping vs Embedding with Link Protection in Filterless Optical Networks
}

\author{
Omran Ayoub*, Leila Askari, Andrea Bovio, Francesco Musumeci and Massimo Tornatore \\ Politecnico di Milano, Milan, Italy.*Corresponding author: omran.ayoub@polimi.it
}

\begin{abstract}
In Filterless Optical Networks (FONs), passive splitters and combiners replace the more complex and more expensive Reconfigurable Optical Add-Drop Multiplexers (ROADMs) based on Wavelength Selective Switch (WSS) in network nodes. The utilization of passive switching elements in network nodes requires to subdivide the network topology into loop-free edge-disjoint fiber trees, consequently constraining physical paths between nodes. In this work, we investigate Virtual Network Mapping (VNM) and Virtual Network Embedding (VNE) with virtual link protection in the context of FONs. To ensure virtual link protection, we propose a strategy based on the placement of additional network equipment to allow mapping virtual links over two fiber trees. We model the problem as an Integer Linear Programming (ILP) formulation with the objective of minimizing network cost in terms of additional network equipment and overall wavelength consumption. Main results show that, when solving VNE, placement of virtual nodes and link mapping can be optimized to avert excessive wavelength consumption, while VNM drains much more network's capacity, since virtual nodes' locations are pre-determined. In addition, results show that, when guaranteeing protection of virtual links in FONs, the gain of VNE is further maximized with respect to VNM. Moreover, results show that solving VNM with virtual link protection requires additional network equipment, severely penalizing network cost.

Index Terms-Filterless Optical Networks, Virtual Network Mapping, Virtual Network Embedding.
\end{abstract}

\section{INTRODUCTION}

Filterless Optical Networks (FONs) are emerging as a promising technical solution to upgrade the capacity in the Wavelength Division Multiplexing (WDM) layer while averting excessive costs. In FONs, the cost of WDM layer components is reduced by replacing expensive active-switching devices, as Reconfigurable Optical Add-Drop multiplexers (ROADMs) based on Wavelength Selective Switches (WSS), by passive optical splitters and combiners that operate on the entire fiber spectrum, thus enforcing a broadcast-and-select node architecture [1].

Due to the absence of filters, FONs must be designed respecting specific criteria to avoid undesired laser-loop effects [2]-[4]. In particular, the network topology must be divided in loop-free fiber trees. The associated design process, called fiber tree establishment, consists in physically interconnecting splitter and combiners such that the physical network is separated into multiple loop-free network segments (i.e., the fiber trees). While fiber trees allow to contain signal broadcasting inside them, they also result in much higher spectrum

Research leading to these results has received funding from the European Commission for METRO-HAUL project (G.A. 761727) and was supported by the National Science Foundation grant 1818972 . consumption with respect to state-of-the-art ROADM-based optical networks (referred in this paper as active optical networks). Moreover, fiber trees constrain the routing and make traditional routing and wavelength assignment problems more challenging. In this work, we study the effect of FON constraints on two such traditional problems as Virtual Network Mapping (VNM), i.e., the assignment of physical resources to virtual links between pre-determined nodes (the placement of virtual nodes over physical nodes is a given of the problem), and Virtual Network Embedding (VNE), i.e., the placement of virtual nodes over physical nodes solved together with the assignment of physical resources to virtual links. In particular, we investigate VNM and VNE considering virtual link protection. However, due to the constraints the fiber tree establishment impose on optical connectivity between nodes, solution to disjoint link protection is infeasible, unless additional network equipment (e.g., transceivers or wavelength blockers) is deployed to guarantee multiple optical routes between nodes. To this end, we propose a strategy based on the placement of additional transceivers to allow mapping virtual links over two fiber trees.

We will study the impact of FONs constraints on VNM and VNE with virtual link protection on network cost, expressed by additional network equipment and wavelength consumption. The problems of VNM and VNE in the context of FONs encompass a number of research questions that, to the best of our knowledge, have not been investigated so far. More specifically: 1) How to perform VNM and VNE (with and without virtual link protection) in FONs? 2) What is the impact of FON constraints on VNM and VNE? 3) Is the difference in wavelength consumption between VNM and VNE in FONs the same as that in active optical networks or higher? In this paper we address these questions by modeling the VNM and VNE with virtual link protection problems through an Integer Linear Programming (ILP) formulation and conducting a thorough numerical analysis.

\section{A. Related Work}

Early works on FONs concentrated on jointly optimizing the fiber tree establishment and the routing and wavelength (or spectrum) assignment (RWA) to minimize overall spectrum consumption such as Ref. [2], where authors provided a design tool based on genetic algorithm, and Ref. [3], where authors proposed a multi-goal evolutionary Pareto optimization considering maximization the demands' protection ratio. Ref. [5] proposed ILP model to perform fiber tree establishment and 
routing and wavelength assignment. In our study, we assume the fiber tree establishment is an input to the problem, (as optimizing the design of a FON only for a set of VNs is not considered a practical objective). Ref. [6], proposed ILP model for resource allocation to minimize spectrum consumption in elastic FONs. With respect to all these approaches, in this study we consider the embedding of virtual nodes, which leads to significant differences in solving the problem compared to traditional RWA and/or VNM. Note that VNE in FON exhibits notable differences with respect to VNE in active optical networks, mainly due to the constraints that fiber trees impose on routing, and, therefore, on mapping of virtual links.

More recent works, such as, [7]- [8], focused on the use of programmable optical switches to limit spectrum waste, proposing ILP models and heuristic approaches for their designs. Results show that overall resource consumption can be greatly reduced for paying off an acceptable additional cost for filtering elements. In our work, we do not assume the placement of filters as the objective of our analysis is to investigate the impact of VNM and VNE on pure FONs and leaving consideration for semi-filterless networks as future work. Protection and survivability aspects in FONs have been studied in [10] and [11], where authors propose a heuristic approach to guarantee $1+1$ optical-layer protection of traffic demands and an ILP model to perform survivable VNM, respectively. Results show that additional network equipment, such as wavelength blockers, must be placed at selected network nodes. Similarly, in our analysis, we consider the placement of additional inter-tree transceivers to allow transfer of the optical signal from one fiber tree to another to enable protection of VNs. To the best of our knowledge, no previous work investigated the embedding of virtual networks and its impact in the context of FONs.

\section{B. Paper Contribution and Organization}

The main contributions of this paper are as follows: (1) we provide formal definition for the problems of VNM and VNE in FON; (2) we propose a strategy for guaranteeing virtual link protection of VNs based on placing additional transceivers, which allow mapping a virtual link over two fiber trees; (3) we propose an ILP formulation to perform VNM and VNE (with and without virtual link protection) while minimizing network cost, expressed in terms of additional network equipment and overall wavelength consumption; (4) we conduct numerical analysis to evaluate VNM and VNE in FONs and highlight the differences they exhibit with respect to VNM and VNE in active optical networks.

The rest of the paper is organized as follows. Sec. II discusses examples of VNM and VNE in FONs emphasizing on main difference with respect to active optical networks. Sec. III states the VNE with link protection problem in FON and proposes the ILP model to solve it. Sec. IV discusses illustrative numerical results and Sec. V draws the conclusion.

\section{VNM AND VNE IN FONS}

Example of VNM in FONs: In Fig. 1 we highlight the effect of the FON's design on VNM. We map the VN (i.e.,

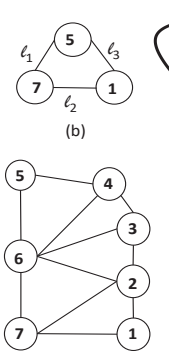

(a)

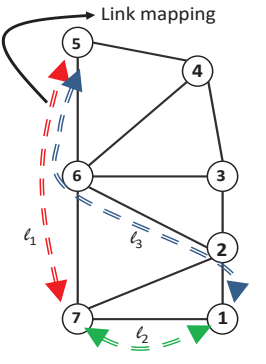

(c)

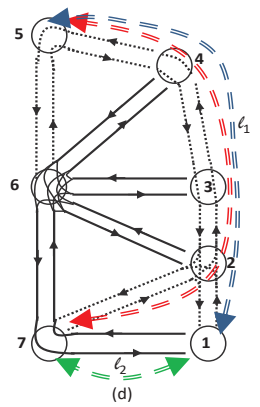

Fig. 1: (a) 7-node network, (b) virtual network, the VNM of the virtual network onto (c) an active optical network and (d) FON with two fiber trees (highlighted by solid and dotted lines).

we allocate a physical optical path to each virtual link) shown in Fig. 1(b) onto the physical network shown in Fig. 1(a) considering active optical network architecture (Fig. 1(c)) and FON architecture (Fig. 1(d)). For the FON, two edge-disjoint fiber trees are considered and are shown with solid and dotted lines, respectively. First, we see that the mapping of the virtual network in the FON significantly differs from that in the active network (two out of three virtual links, $l_{1}$ and $l_{3}$, are mapped differently) because, in FONs, a lightpath cannot transparently traverse fiber trees. For example, virtual link $l_{1}$ cannot be routed through the physical path $(7,6),(6,5)$. Moreover, VNM over FON consumes more wavelength channels (total of $25^{1}$ ) with respect to the VNM in the active network (total of 12) architecture due to i) the longer paths between nodes, and ii) the broadcast-and-select approach employed.

Example of VNE in FONs: In Fig. 2 we highlight the importance of optimizing the VNE in FON and its impact on overall wavelength consumption. We map the VN (one wavelength requested per virtual link) of Fig. 2(a) considering the candidate physical node locations for each of the virtual nodes shown in Fig. 2(b) in three different ways: i) over an active optical network (Fig. 2(c)); ii) over a FON, using the same VNE as that of the active network, i.e., not optimized, (Fig. 2(d)); over FON architecture using the same node embedding as that of active network, but an optimized routing and wavelength assignment (Fig. 2(e)); over a FON architecture with optimized VNE (Fig. 2(f)). For each FON, we consider two fiber trees distinguishable by solid and dotted lines. Moreover, we show by dashed lines the propagation of wavelength on unintended links due to the broadcast architecture in FONs.

First, we see how employing the same VNE of the active network in a FON (2(d)) leads to an excessive wavelength consumption (21 wavelengths instead of 8 occupied in the active network architecture case). When optimizing the routing and wavelength assignment in a FON, but keeping same node embedding as that of active (2(e)), the number of wavelengths occupied decreases to 17 . This is because in our approach (as we will see later), in addition to deciding which physical path to allocate for a virtual link, we optimize

\footnotetext{
${ }^{1}$ We consider one wavelength is requested per virtual link per direction (total of 18 wavelength channels) and wasted channels propagation beyond destination (7 wavelength channels).
} 


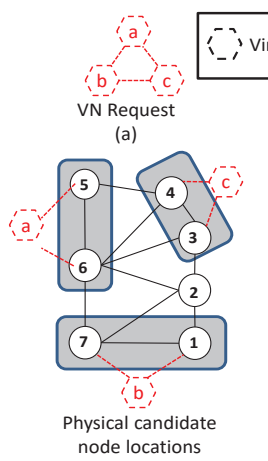

(b)

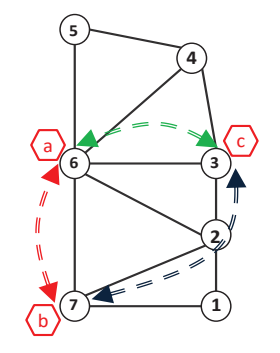

(c)

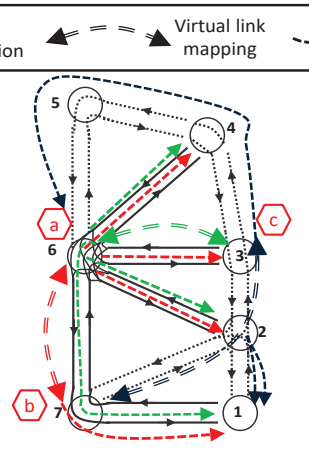

(d)

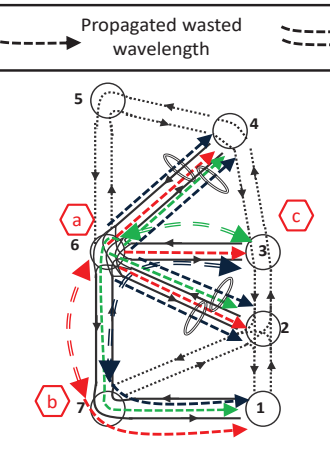

(e)

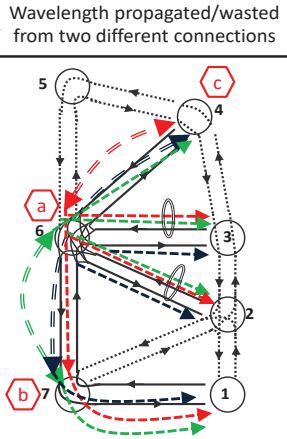

(f)

Fig. 2: (a) VN request, (b) 7-node physical network highlighting candidate physical locations of the virtual nodes of the VN request, (c) Optimized VNE in active optical network, (d) non-optimized VNE over a FON with same mapping of the VN request as in the active optical network, (e) non-optimized VNE over a FON with same virtual node embedding as in the active optical network but optimized RWA, and (f) optimized VNE. Note that the fiber tree establishment considered for FON is the same as that in Fig. 1(b).

the wavelength assignment to virtual links such as to waste the same propagated wavelength on unintended links, which further reduces wavelength consumption. We refer to this aspect by harmless interference and we highlight it in Fig. 2 with an oval shape combining two arrows indicating a wasted wavelength from two different virtual links. Moreover, in Fig. 2(f), we show an optimized VNE in the FON, which further reduces overall wavelength consumption to 16 wavelengths. This suggests that, when performing VNM (and VNE) in FONs, an accurate mapping (and embedding) is crucial to minimize overall wavelength consumption, specially when number of VNs to map (embed) increases.

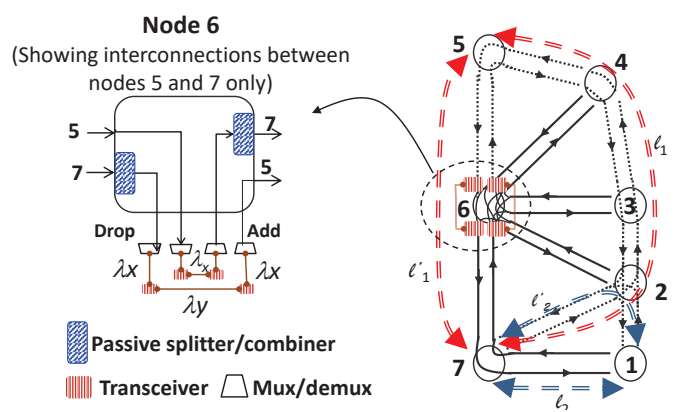

Fig. 3: Example of inter-tree transceiver deployment to allow mapping a virtual link over a physical path on two fiber trees.

Virtual link protection in FONs: Due to the constraints imposed by the fiber tree establishment, protection of virtuallinks, in some cases, is not feasible, as two disjoint physical paths may not exist between two physical nodes. In this context, the deployment of additional network equipment, (for example inter-tree transceivers ${ }^{2}$ ), is mandatory to allow transferring a signal from one fiber tree to another so that a virtual link is mapped over a disjoint physical path. Fig. 3 shows an example of virtual-link protection for two virtual links, $l 1$ and $l 2$. For $l 1(5,7)$, only the working path can be mapped without the use of inter-tree transceivers, while the backup path requires the placement of inter-tree transceivers

\footnotetext{
${ }^{2}$ Other equipment could be used, as wavelength blocker, but the semifilterless case is left as future work.
}

at node 6 to map it over links $(7,6)$ and $(6,5)$ which belong to two different fiber trees. On the contrary, two physical paths exist to allow mapping and protecting $l 2(7,1)$. Note that, mapping a virtual link on two fiber trees means the propagation of its corresponding wavelength onto the two fiber trees, which can negatively affect overall wavelength consumption.

TABLE I: Parameters and sets description for the ILP model

\begin{tabular}{|ll|}
\hline Sets & Description \\
\hline$N$ & Set of physical nodes \\
$E$ & Set of physical links \\
$V$ & Set of virtual networks \\
$S$ & Set of virtual nodes \\
$B$ & Set of virtual links \\
$F$ & Set of filterless fiber trees \\
$G_{f \in F}$ & Set of physical links belonging to fiber tree $\mathrm{f}$ \\
$\tau_{n}$ & Set of candidate physical nodes that a \\
& logical node $\mathrm{n}$ can be mapped to \\
$L$ & Set of wavelengths \\
\hline
\end{tabular}

\section{OPtIMAL VNE WITH LINK PROTECTION IN FONS}

\section{A. Problem Statement}

We model the VNE problem with virtual link protection in FON as an ILP optimization model, referred to as $\boldsymbol{V N E}$ with virtual link protection in FONs (P-VNE-FON). The problem is stated as follows: Given a physical FON topology and a fiber tree establishment, bidirectional links with one fiber per direction and a capacity of $L$ wavelengths, and a set of $\mathrm{VN}$ requests ${ }^{3}$, we decide the embedding of VNs into the FON, (i.e., the placement of virtual nodes in candidate physical node locations and the routing and wavelength assignment of corresponding virtual links, each with disjoint primary and backup physical paths) with the objective of minimizing the overall network cost in terms of additional network equipment and overall wavelength consumption constrained by: $i$ ) nodeembedding, ii) link-mapping, flow and virtual link protection constraints, iii) wavelength continuity and contiguity and iv) maximum physical link capacity constraints. Note that compared to classical networks, we had to re-adapt the flow

\footnotetext{
${ }^{3}$ we assume that each virtual link represents a bidirectional lightpath requests of exactly one wavelength per direction.
} 
TABLE II: Variables description for the ILP model

\begin{tabular}{|lll|}
\hline Variable & Domain & Description \\
\hline$z_{j, s} \in\{0,1\}$ & $j \in N$ & Equal to 1 iff virtual node $s$ is \\
& $s \in S$ & mapped to physical node $j$ \\
$q_{i j}^{s t} \in\{0,1\}$ & $(i, j) \in E$ & Equal to 1 iff virtual link $(s, t)$ \\
& $(s, t) \in B$ & is mapped over physical link $(i, j)$ \\
$w_{i j}^{s t l} \in\{0,1\}$ & $(i, j) \in E$ & Equal to 1 iff wavelength $l$ is \\
& $(s, t) \in B$ & assigned to virtual link $(s, t)$ which \\
& $l \in L$ & is mapped over physical link $(i, j)$ \\
$h_{j i j}^{s t} \in\{0,1\}$ & $j \in N$ & Equal to 1 iff a transceiver \\
& $(i, j) \in E$ & is needed at node j (direction $(i, j))$ \\
& $(s, t) \in B$ & to map virtual link $(s, t)$ \\
$v_{s t}^{l f} \in\{0,1\}$ & $(s, t) \in B$ & Equal to 1 iff virtual \\
& $l \in L$ & link $(s, t)$ uses wavelength $l$ on \\
& $f \in F$ & fiber tree $f$ \\
$p_{i j}^{l} \in\{0,1\}$ & $(i, j) \in E$ & Equal to 1 iff wavelength $l$ is an \\
& $l \in L$ & unfiltered channel on $(i, j)$ \\
$d_{i j}^{j k s t} \in\{0,1\}$ & $(i, j) \in E$ & Equal to 1 iff links $(i, j)$ \\
& $(j, k) \in E$ & and ( $j, k)$ belong to different trees \\
& $(s, t) \in B$ & and they both map virtual link $(s, t)$ \\
$m_{i j j n}^{s t l} \in\{0,1\}$ & $(i, j) \in E$ & Equal to 1 iff $(i, j)$ and $(j, k)$ \\
& $(j, n) \in E$ & map virtual link $(s, t)$ which is \\
& $(s, t) \in B$ & assigned wavelengths $l$ \\
$c_{l} \in\{0,1\}$ & $l \in L$ & \\
& $l \in L$ & Equal to 1 iff wavelength $l$ is used \\
& &
\end{tabular}

constraints to consider wavelength broadcast due to signal splitting in downward direction of a filterless fiber tree.

\section{B. Integer Linear Programming Model}

Set and parameters, and variables are described in Tables I and II, respectively.

Objective function (1): Minimize number of additional intertree transceivers and total wavelength consumption, i.e., the sum of all wavelength channels utilized over all the links including unfiltered wasted wavelength channels (represented by two terms).

$$
\begin{gathered}
\text { Minimize } \sum_{o \in N} \sum_{(i, j) \in E} \sum_{(s, t) \in B} M \cdot h_{o i j}^{s t}+\sum_{(i, j) \in E} \sum_{(s, t) \in B} q_{i j}^{s t} \\
+\sum_{(i, j) \in A} \sum_{l \in L} p_{i j}^{l}
\end{gathered}
$$

Subject to: Virtual node embedding: Constraint 2 ensures that each virtual node is exactly mapped to a physical node and that a physical node can host a maximum of one virtual node for a given $\mathrm{VN}$.

$$
\sum_{i \in \tau_{s}} z_{i s}=1 \quad \forall s \in S
$$

Virtual link mapping and protection: Constraint 3 is the link mapping constraint. It ensures that every virtual link (s,t) of the virtual network is mapped over two physical paths of the physical topology, while constraint 4 ensures that a virtual link uses a maximum of one wavelength per physical link such as to guarantee disjoint physical paths. For the case without protection, constraint 3 is modified such that a virtual link is mapped exactly once. Constraint 5 guarantees that virtual links in both directions follow the same physical path while constraint 6 avoids the self loops during virtual link mapping.

$$
\begin{aligned}
& \sum_{j:(i, j) \in E} q_{i j}^{s t}-\sum_{j:(j, i) \in E} q_{j i}^{s t}=2 \cdot\left(z_{i, s}-z_{i, t}\right) \quad \forall(s, t) \in B, i \in N \\
& q_{i j}^{s t} \leq 1 \quad \forall(s, t) \in B,(i, j) \in E \\
& q_{i j}^{s t}-q_{j i}^{t s}=0 \forall(s, t) \in B,(i, j) \in E \\
& q_{i j}^{s t}+q_{j i}^{s t} \leq 1 \forall(s, t) \in B,(i, j) \in E
\end{aligned}
$$

Wavelength assignment: Constraint 7 ensures that a virtual link uses (for primary and backup) at least two wavelength channels. For the case without protection, constraint 7 is modified such that a virtual link is assigned exactly one wavelength channel. Constraints 8-10 ensure consistency between wavelength assignment and link mapping. Constraint 11 makes sure that two different virtual links cannot be assigned the same wavelength on a physical link.

$$
\begin{gathered}
\sum_{l \in L} \sum_{f \in F} v_{s t}^{l f} \geq 2 \quad \forall(s, t) \in B \\
w_{i j}^{s t l} \leq v_{s t}^{l f} \quad \forall f \in F,(i, j) \in G_{f},(s, t) \in B, l \in L \\
w_{i j}^{s t l} \leq q_{i j}^{s t} \quad \forall f \in F,(i, j) \in G_{f},(s, t) \in B, l \in L \\
w_{i j}^{s t l} \geq v_{s t}^{l f}+q_{i j}^{s t}-1 \quad \forall f \in F,(i, j) \in G_{f}, \\
\quad(s, t) \in B, l \in L \\
w_{i j}^{s t l}+w_{i j}^{u r l} \leq 1 \quad \begin{array}{r}
\forall(i, j) \in E,(s, t) \in B, \\
(u, r) \in B \neq(s, t), l \in L
\end{array}
\end{gathered}
$$

Transceiver placement constraints: Constraints 12-13 identify the node where the deployment of inter-tree transceiver is required. This node represents the node where a virtual link crosses two fiber trees, i.e., is mapped on a physical path crossing two fiber trees.

$$
\begin{gathered}
0 \leq q_{i j}^{s t}+q_{j o}^{s t}-2 \cdot d_{i j j o}^{s t} \leq 1 \quad \forall f \in T, r \in T,(s, t) \in B, \\
(i, j) \in G_{r},(j, o) \in G_{f}: i \neq o, r \neq f \\
h_{j i j}^{s t}, h_{j j o}^{s t} \geq d_{i j j o}^{s t} \quad \forall(s, t) \in B, r \in T, f \in T \\
(i, j) \in G_{r},(j, o) \in G_{f}: r \neq f
\end{gathered}
$$

Wavelength propagation due to broadcast: Constraints 1417 ensure that a wavelength is broadcast on all outgoing ports when passing a filterless node along a fiber tree.

$$
\begin{gathered}
0 \leq w_{i j}^{s t l}+w_{j n}^{s t l}-2 \cdot m_{i j j n}^{s t l} \leq 1 \quad \forall f \in F, l \in L, \\
(s, t) \in B,(j, n) \in G_{f},(i, j) \in G_{f} \neq(j, n) \\
p_{j u}^{l} \geq m_{i j j n}^{s t l} \quad \forall f \in T, l \in L,(s, t) \in B,(i, j) \in G_{f}, \\
(j, n) \in G_{f} \neq(i, j),(j, u) \in G_{f} \neq(i, j),(j, n) \\
p_{j u}^{l} \geq p_{i j}^{l} \quad \forall f \in F, l \in L,(i, j) \in G_{f}, \\
(j, u) \in G_{f}: u \neq i
\end{gathered}
$$




$$
\begin{gathered}
p_{j u}^{l} \geq w_{i j}^{s t l}-1+z_{j t} \quad \forall f \in F, l \in L, \\
(s, t) \in B,(i, j) \in G_{f},(j, u) \in G_{f}: u \neq i
\end{gathered}
$$

Moreover, constraint 18 ensures that there is no overlap in any wavelength used to map virtual links on a physical link. Note that, the constraints allow the same wavelength to be broadcast (wasted) on a physical link, as shown in Fig. 2. Constraints 19 and 20 ensure that if a virtual link $(s, t)$ crosses different fiber trees, each of the wavelength channels assigned on a fiber tree is propagated in the downward direction.

$$
\begin{gathered}
w_{i j}^{s t l}+p_{i j}^{l} \leq 1 \forall(i, j) \in E,(s, t) \in B, l \in L \\
p_{j u}^{l} \geq m_{i j j n}^{s t l} \forall f \in F, l \in L,(s, t) \in B, \\
r \in F,(i, j) \in G_{r},(j, n) \in G_{f}, \\
(j, u) \in G_{r} \neq(i, j): r \neq f \\
0 \leq w_{i j}^{s t l}+d_{i j}^{j n s t}-2 \cdot m_{i j j n}^{s t l} \leq 1 \quad \forall f \in F, l \in L,(s, t) \\
\in B, r \in F,(j, n) \in G_{f} \neq(i, j),(i, j) \in G_{r}, r \neq f
\end{gathered}
$$

\section{Illustrative NumericAl Results}

In this numerical section, we compare all the cases summarized in Tab. III, namely, we consider VNE and VNM, with and without protection, in active and FON networks, resulting in eight different scenarios. Note that, in VNM, the physical node where each virtual node is mapped is part of the problem inputs. Comparing these eight scenarios allows to assess i) the benefits of having more freedom in embedding the virtual nodes (embedding vs mapping), ii) if the impact of VNM vs. VNE is the same in FON as in active networks, and iii) the cost of protection. Note also that the case of active optical networks will serve as benchmark scenario.

\section{A. Evaluation settings}

We use CPLEX 12.10 over a workstation with Intel(R) Core(TM) i7-7500U CPU (6 cores @ 2.70GHz) processor and $32768 \mathrm{MB}$ of memory to solve our optimization instances over a 7-node network (shown in Fig. 1(a)) and a 10-node network

\begin{tabular}{|c|c|c|c|}
\hline $\begin{array}{c}\text { Network } \\
\text { Architecture }\end{array}$ & Protection? & VNE/VNM & Acronym \\
\hline \multirow{4}{*}{ FON } & \multirow{2}{*}{ Protection } & VNE & P-VNE-FON \\
\hline & & VNM & P-VNM-FON \\
\hline & \multirow{2}{*}{ No Protection } & VNE & VNE-FON \\
\hline & & VNM & VNM-FON \\
\hline \multirow{4}{*}{ Active } & \multirow{2}{*}{ Protection } & VNE & P-VNE-Active \\
\hline & & VNM & P-VNM-Active \\
\hline & \multirow{2}{*}{ No Protection } & VNE & VNE-Active \\
\hline & & VNM & VNM-Active \\
\hline
\end{tabular}
[10], considering a number of VNs ranging from 2 to 10. Each VN consists of 3 virtual nodes. For VNM, a VN is randomly assigned to a physical node as an input to the problem, while for VNE, additionally, two adjacent physical nodes are also considered as candidate locations for the virtual node. To achieve more generalizable results, for each case, we report

TABLE III: Network scenarios considered for the analysis

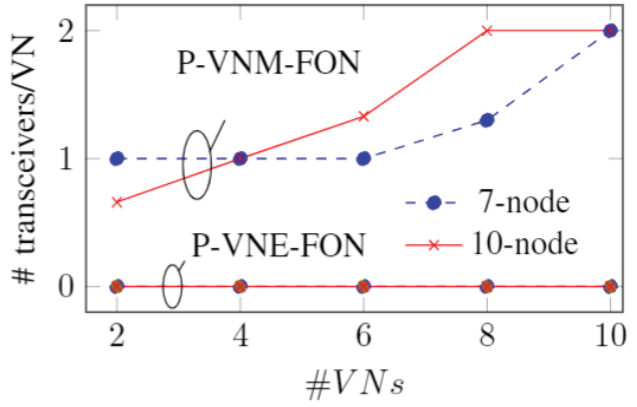

Fig. 4: Number of additional transceivers per VN.

the average of 9 different problem instances resulting from the combinations of three different input VNs and three different fiber tree establishments for each physical network topology. The time required to solve the optimization instances increases with number of VNs and varies between several minutes to an hour for VNM-FON and P-VNM-FON, and between several minutes to 20 hours for VNE-FON and P-VNE-FON.

\section{B. Numerical Results and Discussion}

Additional Inter-Tree Transceivers for Protection: In Fig. 4, we show the additional number of transceivers per VN required to guarantee protection for $P-V N M-F O N$ and $P-V N E$ $F O N^{4}$ for increasing number of VNs and for the 7-node and 10 -node network topologies. For $P-V N E-F O N$, protection is always guaranteed without the need for additional inter-tree transceivers while for $P-V N M-F O N$ the average number of inter-tree transceivers required per $\mathrm{VN}$ ranges between 1 and 2 for the 7-node topology and between 0.8 and 2 for the 10node topology. This shows that when performing VNE, the location of virtual nodes of a virtual link (i.e., the two end points of a virtual link) is optimized such that two disjoint paths belonging to two distinct fiber trees are found between the virtual nodes, hence not requiring the deployment of intertree transceivers. On the contrary, when performing VNM, the deployment of inter-tree transceivers is necessary. This is because, in some cases, such as the case of virtual link $(7,5)$ in Fig. 3, the nodes of a virtual link can optically connect on only one physical path (i.e., on only one fiber tree), and hence, the deployment of inter-tree transceivers is required to guarantee virtual link protection. It is worth noting interesting to note that the placement of inter-tree transceivers contributes further to spectrum waste due to the fact that the wavelength assigned to a virtual link will propagate on two fiber trees.

Wavelength Consumption: Figures 5(a) and (b) show the overall wavelength consumption of the 8 different network scenarios for increasing number of VNs for the 7node and the 10-node physical topologies, respectively. As expected, for all number of VNs, each of FON network scenarios ( $V N E-F O N, V N M-F O N, P-V N E-F O N, P-V N M-F O N)$ consumes more wavelength channels than its active counterpart (VNE-Active, VNM-Active, P-VNE-Active, P-VNM-

\footnotetext{
${ }^{4}$ Note that inter-tree transceivers are only required in a FON, to allow a lightpath to optically cross fiber trees (see Fig. 3)
} 


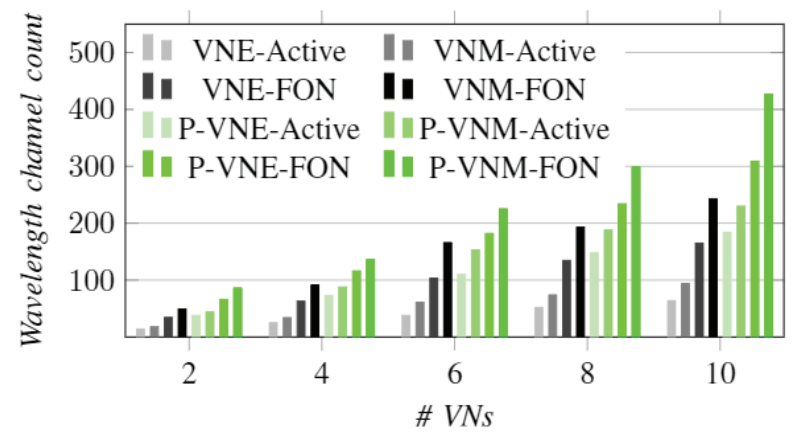

(a) 7-node network

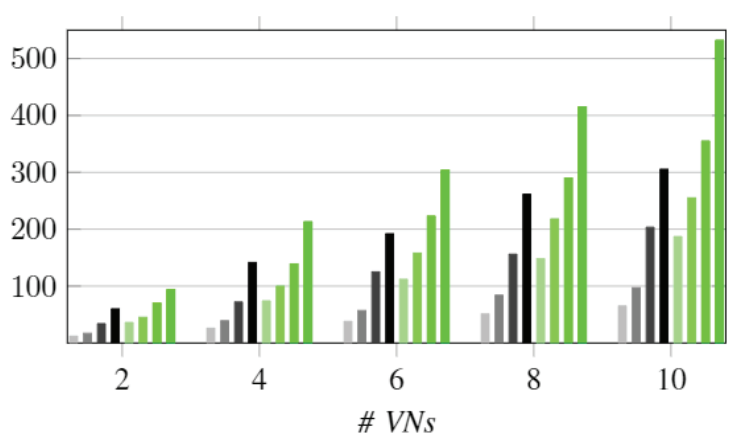

(b) 10-node Network

Fig. 5: Overall wavelength consumption of the eight network scenarios for varying number of VNs in (a) 7-node and (b) 10-node network topologies.

Active). However, when performing VNE, the additional wavelength consumption in FON with respect to active is less than that when performing VNM. For example, VNM-FON consumes 2.6 and 3.5 times more wavelength channels than $V N M$-Active whereas VNE-FON consumes 2.5 and 3.1 times more wavelengths than VNE-Active. Moreover, $P$-VNM-FON consumes 2 times more wavelength channels than $P$-VNMActive while $P$-VNE-FON consumes slightly less than 2 times more wavelength channels than $P$-VNE-Active. This is because when performing VNM, the location of virtual nodes is not optimized according to the fiber tree establishment of the FON, which negatively affects the overall wavelength consumption. On the contrary, when performing VNE, the location of virtual nodes is optimized to cope with the establishment of the fiber trees and avoid excessive spectrum waste.

Let us now concentrate on the effect of introducing protection on the wavelength consumption for FON scenarios. Results show that, while $P$-VNE-FON maintains a steady increase in terms of wavelength consumption (around $15 \%$ to $20 \%$ ), $P$-VNM-FON shows a rapid increase (between $30 \%$ and $50 \%$ ) with the increase in number of VNs to be mapped. This confirms the fact that when performing VNE, the embedding of nodes adapts to the specific filterless tree FONs, and avoids placement of virtual nodes in physical nodes that would consequently affect negatively the wavelength consumption. This also shows that the gain of VNE with respect to VNM is amplified when considering protection in FONs. Additionally, with our approach, through optimizing the VNE, unlike for VNM, the embedding of virtual nodes onto the physical network is jointly optimized with the routing and wavelength assignment, which allows exploiting the harmless interference and further minimizing wavelength consumption.

\section{CONCLUSION}

We investigate the problems of Virtual Network Mapping (VNM) and Virtual Network Embedding (VNE) with virtual link protection in the context of filterless optical networks (FON) and propose a strategy based on placement of additional network equipment (inter-tree transceivers) to allow transferring a signal from one fiber tree to another if required to guarantee protection. We propose an Integer Linear Programming
(ILP) model which performs the mapping and embedding of VNs with the objective of minimizing number of additional network equipment and overall wavelength consumption. Results show that when performing VNE, the embedding of virtual nodes can be adapted to the fiber tree establishment of the FON whereas with VNM, the placement of virtual nodes is decided a-priori, without taking into consideration the specific design aspects of the FON, consequently penalizing network cost in terms of additional equipment and network capacity. As a future work, we plan to develop a scalable heuristic approach and to further extend the work considering virtual node and end-to-end protection.

\section{REFERENCES}

[1] Tremblay, Christine, et al. "Filterless WDM optical core networks based on coherent systems." In proc. International Conference on Transparent Optical Networks, 2011.

[2] Tremblay, Christine, et al. "Agile optical networking: Beyond filtered solutions." In proc. Optical Fiber Communications Conference and Exposition (OFC), 2018.

[3] Krannig, Simon, et al. "How to design an optimized set of fibre-trees for filterless optical networks-The elegance of a multi-goal evolutionary Pareto optimization versus." Photonic Networks; ITG-Symposium; Proceedings of VDE, 2016.

[4] Gunkel, Matthias, et al. "Vendor-interoperable elastic optical interfaces: Standards, experiments, and challenges." IEEE/OSA Journal of Optical Communications and Networking 7.12, B184-B193, 2015.

[5] Jaumard, Brigitte, Yan Wang, and Nicolas Huin. "Optimal design of filterless optical networks." In proc. International Conference on Transparent Optical Networks (ICTON), 2018.

[6] Archambault, Emile, et al. "Routing and spectrum assignment in elastic filterless optical networks." IEEE/ACM Transactions on Networking 24.6, 3578-3592, 2016.

[7] Ayoub, Omran, et al., "Traffic-Adaptive Re-Configuration of Programmable Filterless Optical Networks," IEEE International Conference on Communications (ICC), Dublin, Ireland, 2020.

[8] V. Abedifar and M. Eshghi, "Routing, modulation format, spectrum and core allocation in space-division-multiplexed programmable filterless networks." Optical Fiber Technology 49, 37-49, 2019.

[8] Furdek, Marija, et al. "Programmable filterless network architecture based on optical white boxes." In proc. International Conference on Optical Network Design and Modeling (ONDM), Cartagena, Spain, 2016.

[10] Xu, Zhenyu, et al. " $1+1$ dedicated optical-layer protection strategy for filterless optical networks." IEEE Communications Letters 18(1), pp. 98-101, 2013.

[11] Ayoub, Omran, et al. "Survivable Virtual Network Mapping in Filterless Optical Networks," International Conference on Optical Network Design and Modeling (ONDM), Castelldefels, Barcelona, Spain, 2020. 